\title{
Genetic determinants of glucose levels in pregnancy: genetic risk scores analysis and GWAS in the Norwegian STORK cohort
}

\author{
Gunn-Helen Moen ${ }^{1,2}$, Marissa LeBlanc ${ }^{3}$, Christine Sommer $^{1}$, Rashmi B Prasad $^{4}$, Tove Lekva $^{5}$, \\ Kjersti R Normann 2,6, Elisabeth Qvigstad', Leif Groop ${ }^{4,7}$, Kåre I Birkeland 2,8, David M Evans ${ }^{9,10}$ and \\ Kathrine F Frøslie ${ }^{11}$
}

${ }^{1}$ Department of Endocrinology, Morbid Obesity and Preventive Medicine, Oslo University Hospital, Oslo, Norway, ${ }^{2}$ Institute of Clinical Medicine, Faculty of Medicine, University of Oslo, Oslo, Norway, ${ }^{3}$ Oslo Centre for Biostatistics and Epidemiology, Oslo University Hospital, Oslo, Norway, ${ }^{4}$ Department of Clinical Sciences, Clinical Research Centre, Lund University, Malmö, Sweden, ${ }^{5}$ Research Institute of Internal Medicine, Oslo University Hospital, Oslo, Norway, ${ }^{6}$ Section of Specialized Endocrinology, Department of Endocrinology, Oslo University Hospital, Oslo, Norway, ${ }^{7}$ Finnish Institute of Molecular Medicine (FIMM), Helsinki University, Helsinki, Finland, ${ }^{8}$ Department of Transplantation Medicine, Oslo University Hospital, Oslo, Norway, ${ }^{9}$ University of Queensland, Diamantina Institute, Translational Research Institute, Brisbane, Australia, ${ }^{10}$ Medical Research Council Integrative Epidemiology Unit, University of Bristol, Bristol, UK, and ${ }^{11}$ Norwegian National Advisory Unit on Women's Health, Oslo, Norway

Correspondence should be addressed to G-H Moen Email g.h.o.moen@studmed.uio.no

\begin{abstract}
Objective: Hyperglycaemia during pregnancy increases the risk of adverse health outcomes in mother and child, but the genetic aetiology is scarcely studied. Our aims were to (1) assess the overlapping genetic aetiology between the pregnant and non-pregnant population and (2) assess the importance of genome-wide polygenic contributions to glucose traits during pregnancy, by exploring whether genetic risk scores (GRSs) for fasting glucose (FG), 2-h glucose (2hG), type 2 diabetes (T2D) and BMI in non-pregnant individuals were associated with glucose measures in pregnant women. Methods: We genotyped 529 Norwegian pregnant women and constructed GRS from known genome-wide significant variants and SNPs weakly associated $\left(p>5 \times 10^{-8}\right)$ with FG, 2hG, BMI and T2D from external genome-wide association studies (GWAS) and examined the association between these scores and glucose measures at gestational weeks 14-16 and 30-32. We also performed GWAS of FG, 2hG and shape information from the glucose curve during an oral glucose tolerance test (OGTT).

Results: $\mathrm{GRS}_{\mathrm{FG}}$ explained similar variance during pregnancy as in the non-pregnant population $(\sim 5 \%)$. GRS $\mathrm{BMI}_{\mathrm{B}}$ and $\mathrm{GRS}_{\mathrm{T} 2 \mathrm{D}}$ explained up to $1.3 \%$ of the variation in the glucose traits in pregnancy. If we included variants more weakly associated with these traits, $\mathrm{GRS}_{2 \mathrm{hG}}$ and $\mathrm{GRS}_{\mathrm{T} 2 \mathrm{D}}$ explained up to $2.4 \%$ of the variation in the glucose traits in pregnancy, highlighting the importance of polygenic contributions.

Conclusions: Our results suggest overlap in the genetic aetiology of FG in pregnant and non-pregnant individuals. This was less apparent with 2 hG, suggesting potential differences in postprandial glucose metabolism inside and outside of pregnancy.

\section{Introduction}

Metabolic adaption during pregnancy is important to ensure growth and development of the foetus and to guarantee that the mothers' elevated energy needs during the pregnancy are met (1). Glucose is the major energy source for the foetus, which freely crosses the placenta. Two major changes in glucose metabolism 
take place during pregnancy. First, fasting glucose (FG) levels decrease during early pregnancy, mainly due to an increase in plasma volume (2). Secondly, pregnancy has a diabetogenic effect on the mother, with increased insulin resistance usually commencing after the first trimester. Regardless of the changes in maternal glucose metabolism, and the increase in insulin resistance, maternal fasting blood glucose values normally remain relatively stable throughout pregnancy (1). This is largely because of fetal glucose utilization, although postprandial glucose levels tend to increase (3). However, if the maternal beta cells are not able to compensate for insulin resistance by producing more insulin, the mother runs the risk of hyperglycaemia.

The large, multicentre, Hyperglycaemia and Adverse Pregnancy Outcomes (HAPO) study showed a clear and graded relationship between plasma glucose values during pregnancy and increased disease burden in the mother and offspring $(4,5)$. Hence, hyperglycaemia in pregnancy - even without gestational diabetes (GDM) diagnosis - is associated with increased risk of adverse health outcomes in both mother and child $(6,7)$. Additionally, it has been estimated that around $50 \%$ of the women diagnosed with GDM will develop type 2 diabetes (T2D) within 5-10 years, although the exact risk varies with the criteria used and the follow-up time $(8,9)$. GDM and T2D appear to share both genetic and non-genetic risk factors - such as high BMI - and the overlapping susceptibility may partly explain the increased risk of T2D for women with previous GDM $(10,11)$.

Glucose levels in pregnancy are usually measured in the fasting state or (one and) two hours after an oral glucose tolerance test (OGTT). Commonly, single measurements - such as fasting glucose levels (FG) and $2 \mathrm{~h}$ post OGTT glucose levels ( $2 \mathrm{hG})$ - are used to diagnose GDM. However, different patterns of the glucose levels during an OGTT can yield the same single measurements, and thus, the shape of the glucose curves may provide important additional information. Functional data analysis (FDA) (12) of OGTT glucose curves has previously been used to extract shape information not apparent in commonly used simple measures. Briefly, the most important curve shape characteristic, interpreted as the general glucose level during an OGTT, was positively associated with all OGTT measurements and closely related to the area under the OGTT curve (AUC). The second most important shape characteristic described to what extent the women had a longer than average time to peak and a prolonged time to return to FG levels. The latter curve shape information was found to successfully discriminate between women who did and did not develop GDM later in pregnancy
(13), indicating that the glucose curve shape might hold valuable information of clinical relevance.

Genetic factors are thought to contribute to the increase in blood glucose concentrations during pregnancy. However, whilst more than 100 SNPs have been robustly associated with glycemic traits, hyperglycaemia and T2D in the non-pregnant population $(14,15)$, there are only a few published studies on such associations in pregnant women $(10,11,16,17)$, and it is still not known how well SNPs robustly associated with glucose levels outside of pregnancy (14) are associated in pregnancy.

In the present study, we genotyped up to 529 pregnant women from the Norwegian STORK cohort with glucose phenotypes measured both in the first and third trimester of pregnancy (18). The placenta is assumed to play an important role in the metabolic changes during pregnancy and the two time points for the OGTTs during pregnancy were chosen to reflect the time before the placenta is well established (around gestational week 20), and the time after the placenta is well established and the foetus is gaining weight, in the third trimester. We constructed genetic risk scores (GRSs) of known SNPs for FG, 2hG, BMI and T2D and examined the association with the glucose variables at the two time points during pregnancy, to study the effect of GRSs on the changes in glucose metabolism during pregnancy. In addition to the longitudinal data, the present study introduces the novel approach of combining GRSs with glucose curve shape information in the analyses. We also present genome-wide association studies (GWAS) for FG, $2 \mathrm{hG}$ and shape of the glucose curve during an OGTT. Our aims were to examine the genetic determinants of glucose metabolism during pregnancy, ascertain whether genetic variants robustly associated with glucose metabolism in the non-pregnant population were also associated with the same variables in early and late pregnancy and to see whether known genetic variants associated with $\mathrm{T} 2 \mathrm{D}$ and $\mathrm{BMI}$ were related to glucose variables during pregnancy.

\section{Subjects and methods}

\section{The STORK study}

The STORK study is a prospective cohort of 1031 healthy pregnant women of Scandinavian ancestry who registered for obstetric care at the Oslo University Hospital Rikshospitalet from 2001 to 2008 (18). Exclusion criteria were multiple pregnancy, known history of type 1 or type 2 diabetes mellitus, and severe chronic diseases (pulmonary, cardiac, gastrointestinal or renal). Results 
of a $75 \mathrm{~g}$ OGTT, age, height and weight were recorded at inclusion at gestational weeks (GWs) 14-16. The OGTT was repeated at GW 30-32. This paper includes up to $n=529$ individuals with genotype and repeated clinical and laboratory examinations (Fig. 1). We did not exclude women from the analysis on the basis of GDM diagnosis in pregnancy. The study was approved by the Regional Committee for Medical Research Ethics, Southern Norway, Oslo, Norway (reference number S-2014/224-0119a and S-07392a) and performed according to the Declaration of Helsinki. All participating women provided written informed consent.

\section{Measurements of glucose}

All OGTTs were performed after an overnight fast. Blood samples were taken every $30 \mathrm{~min}$ for $2 \mathrm{~h}$, for a total of five OGTT measurements per woman. Briefly, venous blood

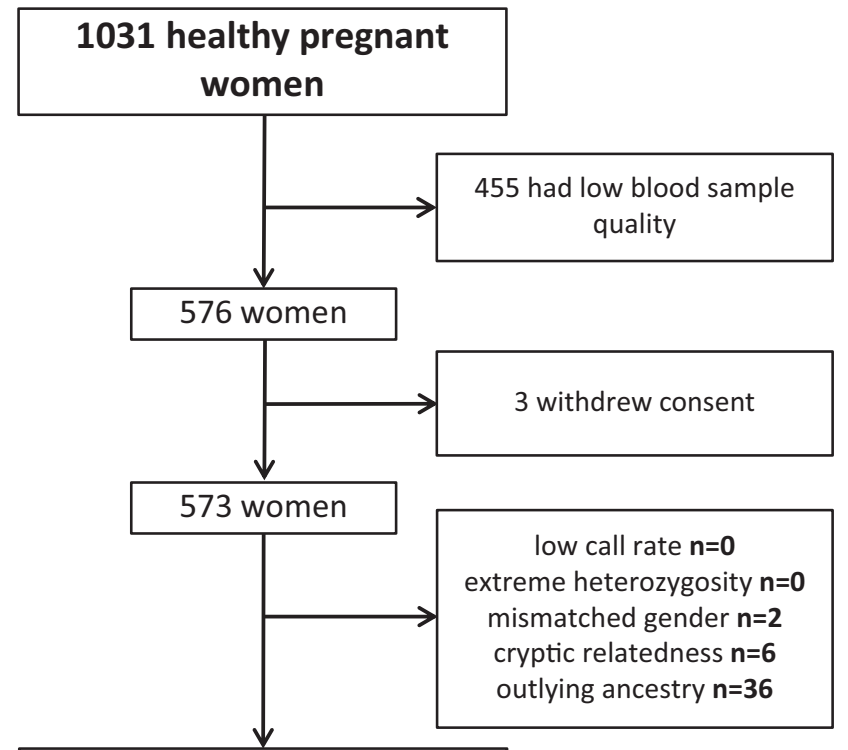

\section{9 healthy pregnant women with DNA}

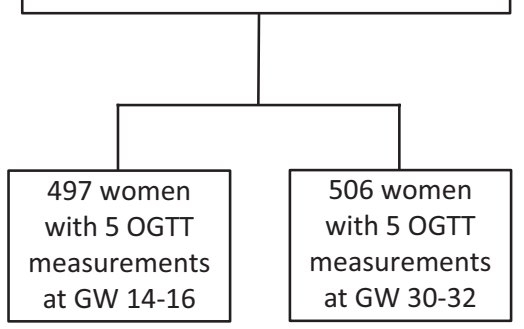

Figure 1

Flowchart for women included in the study. 485 women had 5 OGTT measurements at both GW 14-16 and 30-32. GW, gestational week; OGTT, oral glucose tolerance test. was drawn in gel tubes, allowed to clot for $30 \mathrm{~min}$, and thereafter centrifuged for $10 \mathrm{~min}$ at $1800 \boldsymbol{g}$. Serum was separated and stored at $-80^{\circ} \mathrm{C}$. Glucose was measured from frozen serum samples as previously reported (19) from antenatal visits at GW 14-16 and GW 30-32, with the hexokinase method at an accredited clinical chemistry laboratory at Oslo University Hospital, Rikshospitalet (Cobas 6000 from Roche).

\section{Extracting curve shape information from OGTT glucose curves}

Extraction of curve shape information from OGTT data at GW 14-16 has previously been described in detail (13). In the present study, OGTT data from GW 14-16 and 30-32 were analysed separately. Seven women included in the study were diagnosed with GDM (WHO99 criteria (20)) after GW 14-16; however, they were only treated with dietary advice and they were therefore included in the analysis at GW 30-32. Briefly, the five OGTT measurements from the 497 women in the study sample with genetic data and complete OGTT data at GW 14-16 were converted into 497 continuous, smooth curves (Supplementary Fig. 1A, see section on supplementary data given at the end of this article). The same procedure was used for the 506 women with genetic data and complete OGTT data at GW 30-32 (Supplementary Fig. 1B). A common smoothing parameter was used in both smoothing procedures (21). These 497 and 506 individually fitted curves formed the basis for two separate functional principal component analyses (FPCAs), in which curve shape information was extracted.

In a FPCA, curve shape information is decomposed into a small set (e.g. two) of common principal component (PC) curves, and a corresponding set of individual PC scores, one score per PC per woman. As shown previously (13), the PC curves can be given physiological interpretations according to the temporal variation (shape) they exhibit. A woman's set of individual PC scores reflects how her individual glucose curve shape corresponds to the general temporal feature expressed by the PC curves. Hence, the PC score values from GW 14-16 and 30-32 were used as outcome variables for the shape information inherent in the OGTT glucose curves. FDA (curve fitting and FPCA) was performed using the FDA package in R (https://cran.r-project.org/).

\section{DNA extraction}

At weeks 22-24 and 36-38, peripheral blood mononuclear cells were isolated from venous blood using BD Vacutainer 
CPT Tubes (BD, NJ, USA), centrifuged immediately at $2500 \mathrm{rpm}$ for $25 \mathrm{~min}$, buffycoat separated and transferred carefully to nunc-tubes and stored at $-80^{\circ} \mathrm{C}$ until extraction. DNA was extracted using MagNA Pure Isolation Kit and Instrument (Roche Life Science) at weeks 22-24 and with MagMAX Isolation Kit and Instrument (Applied Biosystems) at weeks 36-38, due to changes in instrumentation at the laboratory over the years. To include as many participants as possible, DNA was also extracted from 291 full blood venous samples stored at $-70^{\circ} \mathrm{C}$ containing EDTA from the follow-up study (19), using a salting out procedure (22) (Miller SA, 1215). Where several DNA samples were available only one was used for genotyping.

\section{Genotyping and genome-wide association analysis}

DNA samples were genotyped on the Illumina Infinium CoreExome chip using Illumina iScan by the Department of Clinical Sciences, Clinical Research Centre, Lund University, Malmö, Sweden. Of the 1031 individuals who provided blood samples, 455 had low quality or low concentration of DNA or withdrew consent $(n=3)$ and could not be analysed further. Of the 573 remaining samples, those with low call rate (i.e. $<95 \%$, $n=0)$, extreme heterozygosity ( $>\mid$ mean $\pm(3 \times$ s.D. $) \mid, n=0)$, mismatched gender $(n=2)$, cryptic relatedness (i.e. one individual (chosen at random) from each related pair, defined as genome-wide IBD >0.185 $(n=6)$ was removed from analysis) or of outlying ancestry (defined by ancestry informative PC analysis based on the variancestandardized relationship matrix generated in PLINK 1.9 software package (23) (https://www.cog-genomics. org/plink/1.9/), $n=36$ ) were excluded from analyses (the ancestry of samples is illustrated in Supplementary Fig. 2 which plots principal coordinates obtained via multidimensional scaling). This left 529 participants with useable SNP data. Variants with call rate $<95 \%$ (14 730 SNPs), out of Hardy-Weinberg equilibrium (exact $P<10^{-6}$, 66 SNPs) or with low minor allele frequency (MAF) $<1 \%$ (254 802 SNPs) were removed before imputation. Quality control was performed using the PLINK 1.9 software package (23). After quality control, 281589 variants were left for imputation. The GWAS scaffold was mapped to NCBI build 37 of the human genome, and imputation to the 1000G reference panel (Phase3- http://www.well. ox.ac.uk/ wrayner/tools/) for European ancestry was performed using IMPUTEv2.3.2 (24). 84609303 SNPs were available after imputation. All SNPs with MAF $<0.02$ and imputation quality $<0.4$ were removed from analysis.
A total of 8717487 SNPs were available for GW 14-16 and 8709903 SNPs were available for GW 30-32.

GWAS analysis of the traits FG, $2 \mathrm{hG}$ and curve shape variables (PC1 and PC2) was performed on the expected allele counts using linear regression assuming an additive genetic model in the software package SNPTEST (25). The QQman R package (26) was used for visualization. Test statistics with $p$ values $\leq 5 \times 10^{-8}$ were considered genomewide significant.

\section{Generating GRSs}

In order to construct GRSs, we used publicly available GWAS data for FG (14) ( $n=46$ 186), 2hG (14) $(n=42854)$, BMI (27) ( $n=339224)$ and T2D (28) $(n=26676$ T2D cases and 132532 controls). We constructed weighted GRS based on the published regression coefficients from the discovery meta-analysis of these papers using the PLINK 1.9 software package (23). We constructed scores using known variants only (i.e. genome-wide significant SNPs from the above papers) and using nominally associated variants selected from across the genome meeting a particular $p$-value threshold (i.e. $p<5 \times 10^{-7} ; p<5 \times 10^{-6}$; $p<5 \times 10^{-5} ; p<5 \times 10^{-4} ; p<0.005 ; p<0.05 ; p<0.1 ; p<0.2$; $p<0.3 ; p<0.4 ; p<0.5 ; p<0.6 ; p<0.7 ; p<0.8 ; p<0.9$ and all $p$ values) in order to capture polygenicity in these traits $(29,30)$. For the known risk scores, if the papers reported several SNPs at one locus, LD was checked and a set of independent (pairwise $r^{2}<0.2$ ) SNPs with the lowest $p$ values were selected to contribute to the scores. Details of the genome-wide significant variants used in construction of the known GRS can be found in Supplementary Tables 1, 2, 3 and 4 .

To evaluate the ability of GRS of known SNPs to explain variation in glycemic related traits in pregnancy, we fitted a multiple regression model that contained a term capturing the effect of the known variants. We also investigated whether polygenicity might help explain variation in our traits of interest. This was done by including an extra term that captured the effect of the GRS of nominally associated variants at the different $p$ value thresholds. In order to guard against the possibility of residual population stratification contaminating our results, we also included covariate terms for the first two ancestry informative PCs in the model. The variance explained by the GRS was quantified by reporting the difference in $r$-squared between the full regression model containing all effects and a nested sub-model containing only the effects of the ancestry informative PCs. Scores yielding $p$ values $\leq 0.05$ in this comparison model were 
considered significant. To account for the 32 statistical tests (4 GRS $\times 8$ outcomes), we also evaluated our results against a Bonferroni corrected $\alpha$ level of 0.0016. All analyses were performed using RStudio, version 1.0.153.

\section{Power calculations}

To estimate the power in our study, we used the genetic power calculator (31). We calculated the power for a GWAS assuming a two tailed type 1 error rate and an $\alpha=5 \times 10^{-8}$. We examined power to detect association at variants known to affect FG measures and power to detect association using GRS. Here we assumed a one tailed type 1 error rate of $\alpha=0.05$ given that we were making a specific directional hypothesis with regards to these known variants. Finally, we estimated the power to detect variation explained by the known GRS assuming a one degree of freedom test with two tails with $\alpha=0.05$.

\section{Results}

\section{Sample characteristics and glucose variables}

Characteristics of the study sample, and glucose measurements at GW $14-16$ and 30-32 are shown in
Table 1. Except for a small difference in age $(p=0.02)$, (i.e. those with available genetic data were slightly older than those without), the clinical characteristics of women with genetic data available were not significantly different from those without genetic data $(0.14 \leq p \leq 0.93)$. The individually fitted OGTT glucose curves at GW 14-16 and 30-32 are shown in Supplementary Fig. 1A and B.

At GW 14-16, results from the FPCA showed that the first two components describing the shape of the glucose curve (PC1 and PC2, Supplementary Fig. 1C and D) explained 89 and $8 \%$ of the temporal variation between the fitted curves, respectively. Similarly, at GW 30-32, results from the FPCA showed that the first two PCs (PC1 and PC2, Supplementary Fig. 1E and F) explained 86 and $10 \%$ of the temporal variation between the fitted curves, respectively. Since PC1 scores were highly correlated with AUC ( $r=0.999$ (GW 14-16) and $r=0.997$ (GW 30-32)) the label 'General glucose level' was chosen for this variable. PC2 scores, on the other hand, corresponded to time of the highest glucose level and the label 'Timing of glucose curve peak' was therefore chosen for this variable. The PCs were labelled according to the physiological information they comprised, based on plots of how an individual curve differed from the mean curve if the corresponding PC scores are low or high (Supplementary Fig. 1C and D) $(12,13)$. Descriptive statistics for the corresponding

Table 1 Descriptive statistics for the study sample and the women in the cohort for whom genetic data were not available. The values presented are mean (s.D.) or frequency (\%) unless otherwise stated.

\begin{tabular}{|c|c|c|}
\hline Sample characteristic & Study sample $(n=529 *)$ & Not in study sample $(n=502 * *)$ \\
\hline Age of mother (years) & $31.6(3.8)$ & $31.0(4.0)$ \\
\hline First time mothers & $270(52 \%)$ & $275(55 \%)$ \\
\hline University education & $458(87 \%)$ & $427(85 \%)$ \\
\hline Daily smokers & $12(2 \%)$ & $10(2 \%)$ \\
\hline Pre-gestational BMI (kg/m²) (self-reported) & $23.8(3.9)$ & $23.5(3.6)$ \\
\hline \multicolumn{3}{|l|}{ OGTT at gestational weeks $14-16$} \\
\hline Fasting glucose $(\mathrm{mmol} / \mathrm{L} ; n=517)$ & $4.6(0.4)$ & $4.6(0.4)$ \\
\hline $2 \mathrm{~h}$ glucose $(\mathrm{mmol} / \mathrm{L} ; n=506)$ & $4.8(1.2)$ & $4.8(1.2)$ \\
\hline PC1, 'General glucose level' ${ }^{\circ}(n=497)$ & $0.0(13.0)$ & - \\
\hline PC2, 'Timing of glucose curve peak' ao $(n=497)$ & $0.0(3.9)$ & - \\
\hline \multicolumn{3}{|l|}{ OGTT at gestational weeks $30-32$} \\
\hline Fasting glucose $(\mathrm{mmol} / \mathrm{L} ; n=521)$ & $4.6(0.4)$ & $4.6(0.5)$ \\
\hline $2 \mathrm{~h}$ glucose $(\mathrm{mmol} / \mathrm{L} ; n=512)$ & $6.2(1.5)$ & $6.2(1.4)$ \\
\hline PC1 scores, 'General glucose level' ${ }^{\circ}(n=506)$ & $0.0(14.7)$ & - \\
\hline PC2 scores, 'Timing of glucose curve peak' ${ }^{a}(n=506)$ & $0.0(4.9)$ & - \\
\hline Weight gain from gestational weeks $14-16$ to $30-32$ & $7.8(2.7)$ & $7.5(2.5)$ \\
\hline Gestational age at birth (weeks) & $40.1(1.6)$ & $39.8(2.0)$ \\
\hline Birth weight $(g)$ & $3615(557)$ & $3560(591)$ \\
\hline
\end{tabular}


PC scores are given in Table 1 . It was noted that neither the mean curves, nor the PC curves at GW 14-16 and 30-32 were identical (Supplementary Fig. 1C, E and D, F, respectively), and the PC scores from the two time points should therefore not be directly compared.

\section{Genetic risk scores}

We found that the GRS for FG $\left(\mathrm{GRS}_{\mathrm{FG}}\right)$ explained a similar amount of variance in FG in pregnancy at both measurement occasions as in the MAGIC consortium data (Table 2 and Supplementary Fig. 7). GRS $_{2 \mathrm{hG}}$ consisting of known variants did not explain $2 \mathrm{hG}$ variation during pregnancy well (Table 2). However, when we included nominally associated variants in construction of the GRS by relaxing the $p$-value threshold for the inclusion of SNPs in the risk score, we explained far larger proportions of the variance in $2 \mathrm{hG}$ during pregnancy, highlighting the importance of polygenic contributions to the trait (Supplementary Fig. 8). A similar trend was seen for $\mathrm{GRS}_{\mathrm{T} 2 \mathrm{D}}$ (Table 2), where known variants explained a small significant proportion of the variance in $2 \mathrm{hG}$ and PC1. This proportion increased with the inclusion of nominally associated SNPs from the T2D GWAS (Supplementary Fig. 9). GRS $_{\text {BMI }}$ explained some of the variance in FG, $2 \mathrm{hG}$ and PC1 (Table 2), and an increase in variance explained was also observed when the $p$ value threshold was lowered (Supplementary Fig. 10). PC2 was not significantly associated with any of the risk scores.

We had between 85 and $>99 \%$ power to detect association (assuming a two tailed $\alpha=0.05$ ) for a GRS that explained the smallest and largest expected proportions of the trait variance (i.e. the GRS of known variants is expected to explain $1.7 \%$ of the variation in $2 \mathrm{hG}$ and $4.8 \%$ of the variance in FG according to the literature (14)). Thus, we are well powered to detect association between our GRS and glucose related traits in pregnancy (should the underlying genetic aetiology of the traits be similar).

\section{Genome-wide association study}

The results from the GWAS analysis can be found in the Supplementary data and in Supplementary Figs 3 and 4. Our main findings are shown in Supplementary Table 5. The results from the look-up of known SNPs are shown in Supplementary Tables 1, 2, 3 and 4. However, our study had low power $(<40 \%)$ to detect single loci that explained $5 \%$ of the phenotypic trait variance at genomewide levels of significance $\left(\alpha=5 \times 10^{-8}\right)$ (power $=1 \%$ for a variant explaining $2 \%$ of the variation). Since it would be very unusual for quantitative trait loci underlying complex traits to explain such large proportions of the phenotypic variance, it follows that we must be cautious in interpreting the results. In the case of our look-up of known glucose variants, we had $49 \%$ power to detect SNPs explaining $0.5 \%$ of the phenotypic variance, and $81 \%$ power to detect variants explaining $1.2 \%$ of the variation in the trait. Previously published GWAS indicate that most of the known loci contributing to glucose-related traits do not explain such large proportions of the phenotypic variance in individuals in the non-pregnant population. Assuming the same in our sample of pregnant individuals suggests that we are also underpowered to detect specific known loci.

Table 2 Phenotypic variance explained (\%) by GRS of known SNPs on outcome variables in GW 14-16 and 30-32. Results are shown as difference in $R^{2}$ between the full regression model containing all effects and a nested sub-model containing only the effect of the ancestry informative principal components.

\begin{tabular}{|c|c|c|c|c|c|c|c|c|}
\hline & \multicolumn{2}{|c|}{$\mathbf{G R S}_{\mathbf{F G}}$} & \multicolumn{2}{|c|}{$\mathbf{G R S}_{\mathbf{2 h G}}$} & \multicolumn{2}{|c|}{$\mathbf{G R S}_{\text {T2D }}$} & \multicolumn{2}{|c|}{$\mathbf{G R S}_{\text {BMI }}$} \\
\hline & $\%$ VE & $p$ value & $\%$ VE & $p$ value & $\%$ VE & $p$ value & $\%$ VE & $p$ value \\
\hline FG GW 14-16 & $4.95^{*}$ & $5.55 \times 10^{-7}$ & 0.02 & 0.779 & 0.05 & 0.636 & 0.36 & 0.181 \\
\hline FG GW 30-32 & $4.01 *$ & $5.09 \times 10^{-6}$ & 0.42 & 0.142 & 0.07 & 0.562 & 1.04 & 0.021 \\
\hline 2hG GW 14-16 & $2.37 *$ & 0.001 & 0.24 & 0.277 & 0.69 & 0.065 & 0.78 & 0.048 \\
\hline 2hG GW 30-32 & 0.90 & 0.033 & 0.46 & 0.128 & 1.31 & 0.010 & 0.37 & 0.171 \\
\hline PC1 GW 14-16 & $2.27 *$ & 0.001 & 0.25 & 0.263 & 1.25 & 0.013 & 0.79 & 0.048 \\
\hline PC1 GW 30-32 & 1.43 & 0.007 & 0.46 & 0.127 & 0.66 & 0.067 & 1.07 & 0.020 \\
\hline PC2 GW 14-16 & 0.00 & 0.917 & 0.01 & 0.853 & 0.00 & 0.904 & 0.08 & 0.531 \\
\hline PC2 GW 30-32 & 0.00 & 0.910 & 0.02 & 0.750 & 0.16 & 0.367 & 0.05 & 0.599 \\
\hline
\end{tabular}




\section{Discussion}

By analysing GRS of known and nominally associated variants, we found that SNPs robustly associated with glucose measurements in the non-pregnant population also explain significant proportions of the variance in the glucose measurements in a cohort of pregnant Norwegian women. Furthermore, we found that some single genetic variants associated with glucose metabolism in the nonpregnant population are also associated with the same measures during pregnancy - although the statistical power for these confirmatory analyses was low.

$\mathrm{GRS}_{\mathrm{FG}}$ explained a similar percentage of variance in FG values during pregnancy as in non-pregnant individuals (14). These finding was consistent in early and late pregnancy and the associations were still significant after Bonferroni correction. GRS $_{\mathrm{FG}}$ also explained a small, but significant percentage of the variation in $2 \mathrm{hG}$ and the general OGTT glucose level (PC1). Due to the strong association between $\mathrm{GRS}_{\mathrm{FG}}$ and both FG and $2 \mathrm{hG}$, and our previous findings of high correlations between PC1 scores and all OGTT glucose measurements (13), the observed effect on PC1 was expected.

Surprisingly, no effects on the glucose phenotypes were found for $\mathrm{GRS}_{2 \mathrm{hG}}$. However, SNPs just beneath the genome-wide significance level (e.g. $p$-value threshold of $<5 \times 10^{-7}$ ) explained more of the variance in this trait. This might suggest that SNPs weakly associated with $2 \mathrm{hG}$ in the non-pregnant population may be more important during pregnancy. Our findings highlight the importance of the polygenic contributions to the trait.

The fact that $\mathrm{GRS}_{\mathrm{FG}}$ explained a similar percentage of variation in the trait both early and late in pregnancy compared to a non-pregnant population, whereas GRS $_{2 \mathrm{hG}}$ explained a negligible (and not significant) proportion of the variation in $2 \mathrm{hG}$ and $\mathrm{PC} 1$ during pregnancy is interesting as we know that FG values normally remain relatively stable throughout pregnancy (1) but that the postprandial glucose levels tend to increase (3). This leads us to speculate that the underlying genetic mechanisms for FG are similar in both pregnant and non-pregnant individuals, but that the mechanisms differ for postprandial glucose levels. More studies to understand the mechanisms for the increase in postprandial glucose levels during pregnancy are therefore important. It is, however, important to note that there are fewer known SNPs associated with $2 \mathrm{hG}$ than FG - explaining less of the variance in the trait and thus we have less power to detect a relationship that might be there. Larger GWAS on $2 \mathrm{hG}$ could reveal more associated SNPs - generating a better GRS - and larger GWAS on pregnant women with OGTT could assist in demonstrating a significant genetic overlap.

$\mathrm{GRS}_{\mathrm{BMI}}$ explained some of the variation in the glucose measurements during pregnancy, suggesting that genetically determined BMI has a significant effect on glucose related variables during pregnancy. This is consistent with the fact that BMI is a shared risk factor for both GDM and T2D $(32,33)$. GRS $_{\text {T2D }}$ explained a significant proportion of the variation in $2 \mathrm{hG}$ and PC1 scores. It should be noted that the associations involving $\mathrm{GRS}_{\mathrm{BMI}}$ and GRS $\mathrm{T}_{\mathrm{T} \text { D }}$ were not significant after Bonferroni correction. Just as for $\mathrm{GRS}_{2 \mathrm{hG}}$, the polygenic GRS based on SNPs with $p$ value thresholds below genome-wide significance explained more variance than the GRS of just the known SNPs alone. As it is the $2 \mathrm{hG}$ value, and not FG, that is being explained by this risk score, this GRS might reflect beta cell function and/or insulin resistance during the OGTT. The involvement of GRS $\mathrm{T}_{\mathrm{T} 2 \mathrm{D}}$ suggests some genetic overlap between GDM and T2D, which is in accordance with previous literature $(10,11)$. One GWAS has investigated the relationship between genotypes and glucose measurements in pregnancy (17) and found genome-wide significant influence of four known T2D genes on FG (Glucokinase Regulator (GCKR), Glucose-6-phosphatase 2 (G6PC2), Protein Phosphatase 1 Regulatory Subunit 3B (PPP1R3B) and Melatonin Receptor 1B (MTNR1B)). A GWAS of GDM, conducted in a Korean population, found that known T2D-associated variants in the CDK5 Regulatory Subunit Associated Protein 1 Like 1 (CDKAL1) and MTNR1B gene regions were also associated with GDM (16). However, although metabolic changes in pregnancy may look similar to those in the development of T2D, and although T2D and GDM share several genetic risk factors, the underlying mechanisms behind the diabetogenic development in pregnancy might be different.

Our study is the first to examine the genetics of OGTT data at several time points during pregnancy. In our GWAS, we found three novel SNPs (rs116745876 (MitogenActivated Protein Kinase Kinase Kinase 1 (MAP3K1)), rs11682804 (Protein Kinase C Epsilon (PRKCE)), rs11112715 (NUAK Family Kinase 1 (NUAK1)), which might potentially be related to glucose metabolism in pregnancy. However, our power calculations strongly suggest that these GWAS findings most likely represent type 1 errors, since the existence of SNPs with large effect size would be very atypical in the case of complex quantitative traits. We will therefore make our single marker test results available for future GWAS meta- 
analyses of these traits, and as a replication resource. The formation of large-scale consortia - as has been done for other glucose-related traits and diseases - will be necessary to understand glucose metabolism during pregnancy and detect novel genetic loci for GDM.

Our look-up of robustly associated glucose-related variants in our study of pregnant mothers showed some nominally significant associations in the expected directions, although most of the SNPs were not significantly associated with the outcomes due to the low power of our study with respect to single-variant associations. When these variants were subsequently combined into GRS, power increased and the combined effects of the SNPs show association with the glucose measures in pregnancy. Finding the expected association between $\mathrm{GRS}_{\mathrm{FG}}$ and FG in early pregnancy confirmed that our sample had utility for using GRSs to test hypotheses about the genetic aetiology of glucose measures during pregnancy. We chose to present our main result without Bonferroni corrections, because the physiological traits the GRS are expected to reflect are highly correlated (13). However, due to the metabolic changes during pregnancy, all analyses might be of physiological interest.

Our previous findings indicated promising clinical relevance of the PC2 scores (13), and the negligible associations between the GRSs and PC2 scores were therefore disappointing. This may partly be explained by the fact that the GRSs in our analyses were all based on single measurements, whereas the dynamic curve feature reflected in the PC2 (a delayed glucose peak combined with a prolonged period of high postprandial values) will not be covered by one single measurement. Similarly, whereas the AUC is a simple and good substitute for the PC1 scores (13), the dynamic, temporal information in the PC2 scores is not as easily replaced by simple measures. Calculating the timing of the glucose peak from five OGTT glucose measurements, without smoothing of the measurements and estimating continuous curves, could result in very crude measurements of the timing of the peak and loss of information and precision. We therefore recommend that this curve characteristic is estimated by FDA. Validation of the physiological relevance of the PCs is important, but challenging, since few studies provide gold standard measurements of metabolic features in combination with FDA of OGTT. In non-pregnant populations, high correlations between $\mathrm{HbA1c}$ and FG and $2 \mathrm{hG}$ have been found $(34,35)$. It may therefore be speculated that the information in average measurements like the HbA1c may partly overlap with the information in the PC1 scores. Furthermore, insulin resistance in the muscle can be shown as impaired glucose uptake after ingestion of a carbohydrate-rich meal and results in postprandial hyperglycaemia (36) and that stress on the pancreatic $\beta$-cells may lead to impaired insulin secretion (37). It is therefore reasonable to speculate that the PC2 might reflect aspects of insulin resistance or secretion. The genetic and environmental contribution to such mechanisms in pregnancy is not known, and these issues should be addressed in future studies, to gain more insight into the dynamics of glucose regulation.

In this study we showed that known SNPs for FG appeared to explain similar percentages of variance in FG at two time points during pregnancy as in the non-pregnant population. GRS for BMI and T2D explained some of the variation in the maternal glucose measurements, including the glucose curve shape component interpreted as the general glucose level. Interestingly, SNPs weakly associated $\left(P>5 \times 10^{-8}\right)$ with $2 \mathrm{hG}$ and T2D in GWAS data from the non-pregnant population seemed to explain more variation in the traits than the known genome-wide significant SNPs alone. These results highlight the importance of polygenic contributions to the glucose traits and suggest an overlap in the genetic aetiology of these traits in pregnant individuals and the non-pregnant population.

\section{Supplementary data}

This is linked to the online version of the paper at https://doi.org/10.1530/ EJE-18-0478.

\section{Declaration of interest}

Kåre I Birkeland is an editor of the European Journal of Endocrinology. The authors declare that there is no conflict of interest that could be perceived as prejudicing the impartiality of this study.

\section{Funding}

This article did not receive any specific grant from any funding agency in the public, commercial or not-for-profit sector. G H M has a PhD grant from the South-Eastern Norway Regional Health Authority (Grant number 2015008). D M E is supported by an NHMRC Senior Research Fellowship (APP1137714). Part of this study was presented at the American Society of Human Genetics conference in Orlando, Florida in October 2017

Author contribution statement

G H M, E Q, K I B, M L, C S, D M E and K F F designed the research; T L, K $\mathrm{R} N$ contributed to data collection and lab analysis; $\mathrm{G} \mathrm{H} \mathrm{M}$ conducted the research and analysed data; G H M, D M E and K F F wrote the paper; $M$ L, C S, R B P, T L, K R N, E Q, L G, K I B contributed to the discussions and edited the paper. All authors read and approved the final manuscript. $K F$ $F$ is the guarantor of this work and, as such, had full access to all the data in the study and takes responsibility for the integrity of the data and the accuracy of the data analysis. 


\section{Acknowledgements}

The glucose analysis was provided by the Department of Medical Biochemistry, an accredited clinical chemistry laboratory at Oslo University Hospital. Data on glycemic traits have been contributed by MAGIC investigators and have been downloaded from www.magicinvestigators. org. Data on T2D have been contributed by the DIAGRAM consortium and have been downloaded from http://diagram-consortium.org. Data on BMI have been contributed by the GIANT consortium and have been downloaded from http://portals.broadinstitute.org/collaboration/giant/ index.php/Main_Page.

\section{References}

1 Hadden DR \& McLaughlin C. Normal and abnormal maternal metabolism during pregnancy. Seminars in Fetal and Neonatal Medicine 200914 66-71. (https://doi.org/10.1016/j.siny.2008.09.004)

2 Metzger BE. The Freinkel legacy. In Textbook of Diabetes and Pregnancy. Eds M Hod, L Jovanovic, GC Di Rienzo, A de Lieva \& O Langer. London: Martin Dunitz, 2003.

3 Froslie KF, Roislien J, Qvigstad E, Godang K, Bollerslev J, Henriksen T $\&$ Veierod MB. Shape information in repeated glucose curves during pregnancy provided significant physiological information for neonatal outcomes. PLOS ONE 20149 e90798. (https://doi. org/10.1371/journal.pone.0090798)

4 HAPO Study Cooperative Research Group, Metzger BE, Lowe LP, Dyer AR, Trimble ER, Chaovarindr U, Coustan DR, Hadden DR, McCance DR, Hod M et al. Hyperglycemia and adverse pregnancy outcomes. New England Journal of Medicine 2008358 1991-2002. (https://doi.org/10.1056/NEJMoa0707943)

5 International Schizophrenia Consortium, Purcell SM, Wray NR, Stone JL, Visscher PM, O’Donovan MC, Sullivan PF \& Sklar P. Common polygenic variation contributes to risk of schizophrenia that overlaps with bipolar disorder. Nature 2009460 748-752. (https://doi.org/10.1038/nature08185)

6 Catalano PM. The impact of gestational diabetes and maternal obesity on the mother and her offspring. Journal of Developmental Origins of Health and Disease 20101 208-215. (https://doi. org/10.1017/S2040174410000115)

7 Gillman MW, Rifas-Shiman S, Berkey CS, Field AE \& Colditz GA Maternal gestational diabetes, birth weight, and adolescent obesity. Pediatrics 2003111 e221-e226. (https://doi.org/10.1542/ peds.111.3.e221)

8 Kim C, Newton KM \& Knopp RH. Gestational diabetes and the incidence of type 2 diabetes. A systematic review. Diabetes Care 2002 25 1862-1868. (https://doi.org/10.2337/diacare.25.10.1862)

9 Damm P, Houshmand-Oeregaard A, Kelstrup L, Lauenborg J, Mathiesen ER \& Clausen TD. Gestational diabetes mellitus and longterm consequences for mother and offspring: a view from Denmark. Diabetologia 201659 1396-1399. (https://doi.org/10.1007/s00125016-3985-5)

10 Watanabe RM. Inherited destiny? Genetics and gestational diabetes mellitus. Genome Medicine 20113 18. (https://doi.org/10.1186/gm232)

11 Lowe WL, Scholtens DM, Sandler V \& Hayes MG. Genetics of gestational diabetes mellitus and maternal metabolism. Current Diabetes Reports 201616 15. (https://doi.org/10.1007/s11892-0150709-z)

12 Ramsay J \& Silverman BW. Functional Data Analysis. New York: Springer, 2005.

13 Froslie KF, Roislien J, Qvigstad E, Godang K, Bollerslev J, Voldner N, Henriksen T \& Veierod MB. Shape information from glucose curves: functional data analysis compared with traditional summary measures. BMC Medical Research Methodology 201313 6. (https://doi. org/10.1186/1471-2288-13-6)
14 Scott RA, Lagou V, Welch RP, Wheeler E, Montasser ME, Luan J, Magi R, Strawbridge RJ, Rehnberg E, Gustafsson S et al. Large-scale association analyses identify new loci influencing glycemic traits and provide insight into the underlying biological pathways. Nature Genetics 201244 991-1005. (https://doi.org/10.1038/ng.2385)

15 Prasad RB \& Groop L. Genetics of type 2 diabetes-pitfalls and possibilities. Genes 20156 87-123. (https://doi.org/10.3390/ genes6010087)

16 Kwak SH, Kim SH, Cho YM, Go MJ, Cho YS, Choi SH, Moon MK, Jung HS, Shin HD, Kang HM et al. A genome-wide association study of gestational diabetes mellitus in Korean women. Diabetes 201261 531-541. (https://doi.org/10.2337/db11-1034)

17 Hayes MG, Urbanek M, Hivert MF, Armstrong LL, Morrison J, Guo C, Lowe LP, Scheftner DA, Pluzhnikov A, Levine DM et al. Identification of HKDC1 and BACE2 as genes influencing glycemic traits during pregnancy through genome-wide association studies. Diabetes 2013 62 3282-3291. (https://doi.org/10.2337/db12-1692)

18 Voldner N. Modifiable Determinants of Newborn Macrosomia and Birth Complications. PhD thesis. University of Oslo, Faculty of Medicine, 2010.

19 Lekva T, Bollerslev J, Godang K, Roland MC, Friis CM, Voldner N, Henriksen $\mathrm{T} \&$ Ueland T. beta-Cell dysfunction in women with previous gestational diabetes is associated with visceral adipose tissue distribution. European Journal of Endocrinology 2015173 63-70. (https://doi.org/10.1530/EJE-15-0153)

20 Buckley BS, Harreiter J, Damm P, Corcoy R, Chico A, Simmons D, Vellinga A \& Dunne F. Gestational diabetes mellitus in Europe: prevalence, current screening practice and barriers to screening. A review. Diabetic Medicine 201229 844-854. (https://doi.org/10.1111/ j.1464-5491.2011.03541.x)

21 Salvatore S, Bramness JG \& Roislien J. Exploring functional data analysis and wavelet principal component analysis on ecstasy (MDMA) wastewater data. BMC Medical Research Methodology 201616 81. (https://doi.org/10.1186/s12874-016-0179-2)

22 Miller SA, Dykes DD \& Polesky HF. A simple salting out procedure for extracting DNA from human nucleated cells. Nucleic Acids Research 198816 1215. (https://doi.org/10.1093/nar/16.3.1215)

23 Purcell S, Neale B, Todd-Brown K, Thomas L, Ferreira Manuel AR, Bender D, Maller J, Sklar P, de Bakker Paul IW, Daly Mark J et al. PLINK: a tool set for whole-genome association and populationbased linkage analyses. American Journal of Human Genetics $2007 \mathbf{8 1}$ 559-575. (https://doi.org/10.1086/519795)

24 Howie BN, Donnelly P \& Marchini J. A flexible and accurate genotype imputation method for the next generation of genomewide association studies. PLoS Genetics 20095 e1000529. (https://doi. org/10.1371/journal.pgen.1000529)

25 Marchini J, Howie B, Myers S, McVean G \& Donnelly P. A new multipoint method for genome-wide association studies by imputation of genotypes. Nature Genetics 200739 906. (https://doi. org/10.1038/ng2088)

26 Turner SD. qqman: an R package for visualizing GWAS results using Q-Q and manhattan plots. bioRxiv 2014.

27 Locke AE, Kahali B, Berndt SI, Justice AE, Pers TH, Day FR, Powell C, Vedantam S, Buchkovich ML, Yang J et al. Genetic studies of body mass index yield new insights for obesity biology. Nature 2015518 197-206. (https://doi.org/10.1038/nature14177)

28 Scott RA, Scott LJ, Magi R, Marullo L, Gaulton KJ, Kaakinen M, Pervjakova N, Pers TH, Johnson AD, Eicher JD et al. An expanded genome-wide association study of type 2 diabetes in europeans. Diabetes 201766 2888-2902. (https://doi.org/10.2337/db16-1253)

29 Evans DM, Brion MJ, Paternoster L, Kemp JP, McMahon G, Munafo M, Whitfield JB, Medland SE, Montgomery GW, Timpson NJ et al. Mining the human phenome using allelic scores that index biological intermediates. PLoS Genetics 20139 e1003919. (https://doi. org/10.1371/journal.pgen.1003919) 
30 Evans DM, Visscher PM \& Wray NR. Harnessing the information contained within genome-wide association studies to improve individual prediction of complex disease risk. Human Molecular Genetics 200918 3525-3531. (https://doi.org/10.1093/hmg/ ddp295)

31 Purcell S, Cherny SS \& Sham PC. Genetic Power Calculator: design of linkage and association genetic mapping studies of complex traits. Bioinformatics 200319 149-150. (https://doi.org/10.1093/ bioinformatics/19.1.149)

32 Kalliala I, Markozannes G, Gunter MJ, Paraskevaidis E, Gabra H, Mitra A, Terzidou V, Bennett P, Martin-Hirsch P, Tsilidis KK et al. Obesity and gynaecological and obstetric conditions: umbrella review of the literature. BMJ 2017359 j4511. (https://doi. org/10.1136/bmj.j4511)

33 Lotta LA, Abbasi A, Sharp SJ, Sahlqvist AS, Waterworth D, Brosnan JM, Scott RA, Langenberg C \& Wareham NJ. Definitions of metabolic health and risk of future type 2 diabetes in BMI categories: a systematic review and network meta-analysis. Diabetes Care 2015 38 2177-2187. (https://doi.org/10.2337/dc15-1218)

34 Bozkaya G, Ozgu E \& Karaca B. The association between estimated average glucose levels and fasting plasma glucose levels. Clinics 2010 65 1077-1080. (https://doi.org/10.1590/S1807-59322010001100003)

35 van 't Riet E, Alssema M, Rijkelijkhuizen JM, Kostense PJ, Nijpels G \& Dekker JM. Relationship between A1C and glucose levels in the general Dutch population: the new Hoorn study. Diabetes Care 2010 33 61-66. (https://doi.org/10.2337/dc09-0677)

36 Ferrannini E, Simonson DC, Katz LD, Reichard G, Bevilacqua S, Barrett EJ, Olsson M \& DeFronzo RA. The disposal of an oral glucose load in patients with non-insulin-dependent diabetes. Metabolism 198837 79-85. (https://doi.org/10.1016/0026-0495(88)90033-9)

37 DeFronzo RA \& Abdul-Ghani M. Assessment and treatment of cardiovascular risk in prediabetes: impaired glucose tolerance and impaired fasting glucose. American Journal of Cardiology 2011108 3B-24B. (https://doi.org/10.1016/j.amjcard.2011.03.013)
Received 8 June 2018

Revised version received 10 August 2018

Accepted 18 September 2018 